\title{
An Energy-Efficient Scheduling Scheme for Time-constrained Tasks in
}

\section{Local Mobile Clouds}

\author{
Ting Shi ${ }^{1}$, Mei Yang ${ }^{1}$, Xiang $\mathrm{Li}^{2}$, Qing Lei ${ }^{3}$, YingtaoJiang ${ }^{1}$ \\ ${ }^{1}$ Department of Electrical and Computer Engineering, University of Nevada, Las Vegas, USA \\ ${ }^{2}$ College of Computer Science and Technology, Harbin Engineering University, China \\ ${ }^{3}$ School of Information Technology \& Management, University of International Business and \\ Economics, China \\ Emails: ting@unlv.nevada.edu, mei.yang@unlv.edu, leexiang@hrbeu.edu.cn, \\ leiqing@uibe.edu.cn, yingtao.jiang@unlv.edu
}

\begin{abstract}
Mobile Cloud Computing (MCC) enables mobile devices to use resource providers other than mobile devices themselves to host the execution of mobile applications. Various mobile cloud architectures and scheduling algorithms have been studied recently. However, how to utilize MCC to enable mobile devices to run complex real-time applications while keeping high energy efficiency remains a challenge. In this paper, firstly, we introduce the local mobile clouds formed by nearby mobile devices and give the mathematical models of the mobile devices and their applications. Secondly, we formulate the scheduling problem in local mobile clouds. After describing the resource discovery scheme and the adaptive, probabilistic scheduling algorithm, we finally validate the performance of the proposed algorithm by simulation experiments.
\end{abstract}

\section{Keywords: Mobile cloud computing, ad-hoc network, offloading, task scheduling.}

\section{Introduction}

Mobile devices become a crucial part of our daily life nowadays. Although there have been many advances in technology, mobile devices, due to the restrictions on weight, size, battery life, and heat dissipation, are more resource-constrained than their non-mobile counterparts. Tremendous expand a single mobile device's limited capabilities, offloading is used in Mobile Cloud Computing (MCC), using resource providers other than the mobile device itself to host the execution of mobile applications. There are two types of mobile cloud architectures nowadays. One is the remote cloud, which is defined as a powerful server or a cluster of computer hardware and software that offer the services to the mobile device through WAN connection. Under this architecture, mobile devices typically access cloud services through the cellular network or access points [1]. However, recent research [2] shows that, the remote cloud is unsuitable for complex real-time applications with real-time constraints due to the high communication latency. Therefore, the other architecture, local mobile clouds formed by nearby mobile devices have been proposed to 
support real-time applications. In this architecture, mobile services access the computing resources through the mobile ad hoc network.

In the local mobile clouds, offloading involves making decisions regarding where to offload the tasks to maximize the benefit of offloading, also known as the task scheduling problem. The offloading decisions are usually constructed by analyzing parameters, including bandwidths, computation speeds, power coefficients, and task queues of all participating devices. One of the benefits of offloading is to meet a stringent response time requirement in mobile systems, particularly for real-time applications such as object recognition [3], disaster forecast [4], real-time video coding [5], and speech recognition [7]. These applications typically involve large computation using small datasets. For example, in real-time moving object recognition and tracking system [6], a robot needs to recognize an object then adjust its speed and direction to track the object's movement. If the robot's processor is too slow, the recognition computation might not be able to be completed before the object moves out of the surveillance range. Another benefit of offloading is to improve energy efficiency on mobile devices. Even though battery technology has been steadily improving, it has not been able to keep up with the rapid growth of energy consumption of these mobile systems. Therefore, offloading is also used to save energy by migrating the computational tasks with high energy consumption to more energy-efficient devices.

Various scheduling algorithms have been proposed to make offloading decisions to reduce task completion time, satisfy quality of service (QoS) requirements, or to save energy [8]-[12]. Generally, the schedulers can be classified into two types: centralized and decentralized. In the centralized scheduler such as in [10], the scheduling decisions are made by a central controller based on the knowledge of all the participating nodes. It suffers from bottleneck, single point failure and unrealistic deployment in mobile environment as introduced in [14]. On the other hand, the decentralized scheduling architecture, where each node makes its own scheduling decision, emerges as a promising approach for local mobile clouds because of its scalability and flexibility.

However, existing decentralized scheduling schemes for local mobile clouds have several crucial limitations, including:

1) Scheduling only for one source mobile device (referred as source node), instead of the entire cloud [4][12].

2) Unrealistically assuming that the detailed resource information of each participating processing mobile device (referred as processing node), such as the task queue length and power coefficients, is known as the source node at all time [2][11].

To solve the aforementioned problems for local mobile clouds, herein we propose an adaptive probabilistic scheduler which aims to schedule tasks from multiple source nodes to nearby 
processing nodes, satisfying the tasks' time constraints while keeping low-energy consumption for real-time applications. The proposed scheduler first estimates the completion time and energy consumption at each participating processing node. Next, it schedules the current task to the energy efficient processing node in a probabilistic way and adaptively adjusts its time margin under the unpredictable network condition. Comparing with other three scheduling schemes in proposed in the literature, the experimental results confirm that the proposed scheduler achieves the highest task completion rate and the lowest average energy per successful task in different scenarios. The major contributions of this paper lie in two folds. First, to the best of our knowledge, this is the first work on task scheduling from multiple source nodes to processing nodes in mobile clouds. Second, the proposed adaptive probabilistic scheduler offers a viable solution for scheduling tasks of real-time applications by employing a practical resource discovery mechanism to discover the up-to-date neighboring computational resources.

The rest of the paper is organized as follows. The related work is reviewed in Section 2. Problem formulation and the solutions are provided in Sections 3 and 4, respectively. In Section 5, the performance results are presented to compare the proposed adaptive probabilistic scheduler with three popular schedulers. Finally, the conclusions are summarized in Section 6.

\section{Related work}

The commonly used scheduling objectives in a mobile cloud computing environment are to reduce the tasks' completion time [6][11] and energy consumption [11][15]-[17]. Different scheduling schemes with more complex criteria are proposed in several other papers. A customizable task scheduler is proposed in [12] for local mobile clouds. It is customizable because the user can optimize multiple objectives such as energy consumption and (or) throughput by adjusting the corresponding coefficients in the objective function. Similar work has been done in [11]. The objective function has two parts: 1) delay which includes the task execution time and queuing time at the processing node and communication time, and 2) energy consumption, which consists of computation energy consumed at the processing node and communication energy along the path. The tasks can be delay constrained, energy constrained or delay/energy constrained.

In [6], a real-time moving object recognition and tracking system is presented. An offloading decision framework is developed to divide the computation between the robot and the server such that the total completion time can be minimized in order to satisfy real-time constraint. The MAUI system [11] enables fine-grained energy-aware offload of mobile code to the cloud aiming to minimize the total energy consumption while satisfying the constraint of execution time. Their evaluation shows that several real-time applications, including face recognition, arcade game, and language translation, achieve significant gains through offloading. A machine learning-based 
runtime scheduler is proposed to make offloading decisions based on previous behaviors and current conditions [18]. The most recent work on task scheduling in mobile clouds [17] aims to minimize the total energy consumed consumption of an application in mobile device under a hard completion time constraint.

A number of energy-aware task allocation schemes have been proposed for mobile ad-hoc networks (MANETs). A distributed task allocation approach is described to assign tasks in a workflow to mobile hosts based their capabilities and their mobility patterns [19]. In [15], the energy-aware task scheduling problem for MANET is first formulated and a static algorithm [20] is proposed to make the most of all available resources in such a cooperative wireless environment. A graph-based approach [21] matches the user task graph and the simplified network services graph to achieve the composed user application. The dynamic task allocation scheme in [22] aims to prolong network lifetime by allocating tasks such that minimum residual energy is maximized in a cluster. The self-adaptive method of task allocation scheme [23] prolongs the lifecycle of a cluster by alleviating the load of the cluster head and balancing the load of nodes. Another task allocation scheme for clustering-based MANET [24] aims to maximize the network residual life and achieves better performance than round-robin schedulers. Noticeably, the overhead of maintaining the network structure in a clustering mobile network is not trivial. As such, our work is focused on non-clustered mobile cloud.

Most of the aforementioned work is only limited to one mobile device connecting to one processing node scenario. In a local mobile cloud system, there may exist multiple source devices and multiple processing nodes. In [27], a decentralized dynamic scheduling approach entitled the community aware scheduling algorithm (CASA) is introduced for mobile grids. The scheduler sends the 'offloading' request containing the task information to nearby nodes. Nearby nodes which are able to execute this task will reply to the source node an 'accept' message with the task response time included, which represents the time elapsed between a task's arrival time and the time when the execution result is received. Considering that multiple requests from different source nodes are generated simultaneously, and all source nodes greedily select the processing node offering the shortest task response time. Then that processing node may receive an imbalanced amount of tasks within one scheduling cycle, which might increase the response time for some received tasks. To mitigate this effect, the CASA scheduler adopts a probabilistic approach wherein the probability that a task is scheduled to certain processing node is proportional to the node's computation ability. Comparing with the greedy based scheduling algorithm [28], CASA can lead to a shorter average task response time. This work gives us the insight of how to handle multiple source devices. 
However, it is not clear how each source node can obtain the real-time resource information from the participating nodes to maximize the offloading benefit. Within the field of local mobile clouds, there are, broadly, two different approaches to discover the nearby resource information: reactive and proactive schemes [25]. Reactive discovery schemes as in [27] request other nodes' information when needed, causing small resource discovery overheads but longer reaction time. On the other hand, proactive discovery schemes collect the resource status of various nodes in the network periodically, thus making scheduling decision faster as the resource information is already present. Proactive discovery schemes are more attractive for time-constrained tasks, though they may cause large resource discovery overhead. In [4], A QoS OLSR [29] based proactive routing scheme is proposed to discover all nearby heterogeneous resources. This work shows the feasibility of providing cloud services in the mobile ad-hoc network. The original QoS OLSR is modified by adding resource information, including CPU type, CPU utilization percentage, the allocated memory and battery levels to control messages. Control messages are broadcasted to the whole network periodically so that all nodes are aware of the resource information about other nodes. However, how frequent the control messages are sent and its effect on the scheduling result is not studied in this paper. In fact, since the resource information is updated upon receiving the control messages, the more frequent, the control messages are, the more accurate the resource information is. A frequent resource information update may bring larger overhead and have a major impact on the local mobile cloud's scheduling results.

In contrast, our work is focused on scheduling for time-constrained tasks aiming to minimize the average energy consumption in the entire local mobile cloud. We propose an adaptive probabilistic scheduler which relies on periodic control messages to discover neighboring computational resources. We will investigate how the resource information update frequency affects the scheduling results in detail in Section 5.

\section{Problem formulation}

\subsection{Notations and assumptions}

Table 1 lists the notations used in this paper.

\section{Table 1. Notations}

\begin{tabular}{cc}
\hline Symbol & Description \\
\hline$V$ & Set of all wireless nodes in the local mobile cloud \\
$E$ & Set of all wireless links in the local mobile cloud \\
$G(V, E)$ & Undirected topology graph that is composed of node set $V$ and edge set $E$ \\
$P$ & Set of all processing nodes in the local mobile cloud, $P \subseteq V$ \\
$S$ & Set of all source nodes in the local mobile cloud, $S \subseteq V$ \\
$J$ & Set of tasks arriving at $V$ \\
\hline
\end{tabular}

Assumptions: 
(1) The tasks in $J$ are assumed to be computationally intensive, mutually independent, and can be executed at any participating processing mobile device (or node). As soon as a task arrives, it must be assigned to one processing node for execution. After a task is executed at the processing node, the result will be returned to the source device.

(2) The size of data $D_{j}$ (in bytes) that needs to be transferred from the source node to the destination node is the same as the size of results returned from the destination node to the source node.

(3) The transmission power $e_{t}$, the receiving power $e_{r}$, and the transmission range $r_{t}$ are assumed to be the same among all the nodes. The maximum bandwidth $B_{\max }$ is shared among adjacent nodes.

(4) All nodes in the local mobile cloud are randomly distributed. Any two nodes are connected either directly or in an ad-hoc way.

\subsection{Network and task models}

\subsubsection{Network model}

Definition 1. A local mobile cloud that consists of a number of wireless nodes can be modeled by an undirected communication graph $G(V, E)$. Given a node $u \in V$ and a node $v \in V$, we have an edge $(u, v) \in E$, if and only if $\operatorname{dis}(u, v) \leq r_{t}$, where $\operatorname{dis}(u, v)$ is the Euclidean distance between node $u$ and node $v$. That is, to establish a direct communication between any two nodes, the distance between them has to be within their radio transmission range, $r_{t}$.

The computational ability of the processing node $u$ is denoted by mips $s_{u}$ (million instructions per second). Historical data [30] suggests that power on modern processors is proportional to the square of the duty cycle. Therefore, in this paper we will assume that the CPU's energy consumption per instruction $\mathrm{e}_{u}$ is proportional to the square of its speed, mips $s_{u}$, that is,

$$
e_{u} \propto \operatorname{mips}_{u}{ }^{2}
$$

In each node, $t_{q, u}$ is the queuing delay, which refers to the waiting time when the task is placed at the end of the queue until the moment that the task is processed. The nodes can be classified into two types according to their computational power in mips: less powerful source nodes which can process their own tasks only, and more powerful processing nodes which can process other nodes' tasks.

\subsubsection{Task model}

For a task $j \in J$ (i.e., the task queue at a source node), it has the following attributes:

1) $D_{j}$, size of total data to be transferred between the source node and the processing node;

2) $C_{j}$, amount of computations to be processed; 
3) $T_{j}$, time constraint of task $j$.

All these attributes of task $j$ are known to the source node when $j$ arrives.

\subsection{Problem statement}

With the network and task models, the energy consumption and completion time can be calculated. The energy consumption of executing a task includes two parts:

1) Computation energy, which is the energy dissipated for executing the task by the processing node $u$;

$$
\text { Computation Energy } \text { E }_{j}=e_{u} \times C_{j}
$$

2) Communication energy, which is the energy consumed in communication for the offloading process. The communication energy dissipated is proportional to the amount of data transmitted or received. $H(j)$ is the hop count from the source node to the processing node.

$$
\text { Communication Energy } \text { En }_{j}=H(j) \times\left(e_{t}+e_{r}\right) \times D_{j}
$$

The total energy Task Energy $y_{j}$ consumed by task $j$ is the summation of these two parts of energy:

$$
\text { Task Energy } \text { E }_{j}=\text { Computation Energy }_{j}+\text { Communication Energy }_{j}
$$

The total energy consumed by all the $n$ tasks is:

$$
\text { Total Energy }=\sum_{j=1}^{n} \text { Task Energy }
$$

The completion time of a task contains three parts:

- $t_{q, u}$ : queuing time at the processing node $u$, which is the waiting time before a task can be executed;

- $t_{\text {execution }}$ : the execution time for the task at the processing node $u$;

$$
t_{\text {execution }}=C_{j} / \text { mips }_{u}
$$

- $t_{\text {transmition }}$ : the time needed to transmit the data between the source and destination nodes.

$$
t_{\text {transmition }}=(H(j)-1) \times D_{j} / B_{u, v}
$$

The completion time of the task $j$ when it is offloaded to node $u$ is the total of the three parts:

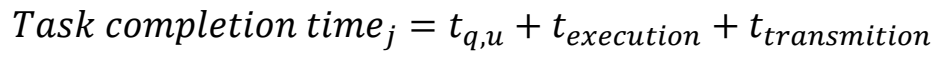

A task is successfully scheduled if it is completed before its deadline, satisfying Eqn. (9); otherwise, it is failed.

$$
\text { Task completion } \text { time }_{j}<T_{j}
$$

Let $n$ be the total number of tasks. We define the following two metrics to evaluate the task scheduling results.

$$
\text { Task Completion Rate }=\text { Number of Successful Tasks } / n
$$


Average Energy per Successful Task = Total Energy/Number of Successful Tasks

The scheduling problem in a local mobile cloud is thus formulated as:

Given: the local mobile cloud $G(V, E)$ with a set of source nodes $S$ and a set of tasks $J$ to be scheduled, schedule these tasks initiated from one or multiple source nodes to processing node(s);

Objective:

Minimize: Average Energy per Successful Task

Subject to: $\quad B_{u, v} \leq B_{\max }, \forall e=(u, v) \in E$,

$$
\sum_{u \in P} x_{j, u}=1, \forall j \in J, x_{j, u} \in(0,1)
$$

$$
P \subseteq V, S \subseteq V, P \cap S=\varnothing, P \cup S=V
$$

Constraint (12) ensures no link capacity is violated. Constraint (13) ensures that a task is scheduled only once. Constraint (14) states that a node in the local mobile cloud is either a processing node or a source node.

\section{Adaptive probabilistic scheduling scheme}

To solve the above task scheduling problem, we propose a distributed adaptive probabilistic scheduler which consists of two phases, namely the resource discovery phase and the adaptive probabilistic scheduling phase. In the resource discovery phase, source nodes collect the resource information of the neighbor processing nodes periodically. In the adaptive probabilistic scheduling phase, at each source node, upon arrival of a task $j$, the scheduler will choose one processing node to execute the task $j$ satisfying the time constraint with lower energy consumption.

\subsection{Phase I: Resource discovery phase}

The proposed resource discovery scheme is based on QoS OLSR [29]. There are two types of control messages carrying resource information:

- Modified Hello Messages, which are sent locally (i.e. broadcasted to one-hop neighbors) to enable a node to discover its local neighborhood (as HELLO messages in the OLSR protocol [31]);

- Modified Topology Control (TC) Messages, which are sent to the entire network through Multipoint Relay (MPR) nodes [31] to allow the distribution of the topology and resource information to all the nodes (as TC messages in the OLSR protocol [31]).

Note that both two types of messages are sent periodically. The emission interval should be a variable related to how fast the network changes. The faster the network changes, the shorter the emission interval should be. According to [31], the emission intervals for the original Hello messages and the original TC messages are $2 \mathrm{~s}$ and $5 \mathrm{~s}$. The emission intervals of the Modified Hello Messages and the Modified TC messages are going to be short considering the tight time 
constraint set by the applications. How the emission intervals affect the scheduling performance in local mobile clouds is to be evaluated in Section 5.

Our scheme is toward an extension of the routing table by adding the following parameters (represented in 4 bytes) to each neighbor ID in the control messages:

- Device parameter: the processing speed mips $s_{u}$ and power coefficient $e_{u}$ of node $u$.

- Queue length: current queue time $t_{q, u}$ at node $u$.

The modified TC Messages sent from an MPR node carry its neighbor node IDs and their resource information. Once receiving the modified control messages, each source node updates its neighbor table, which records the resource information of all participating processing nodes. The distributed resource discovery algorithm is listed in Fig. 1.

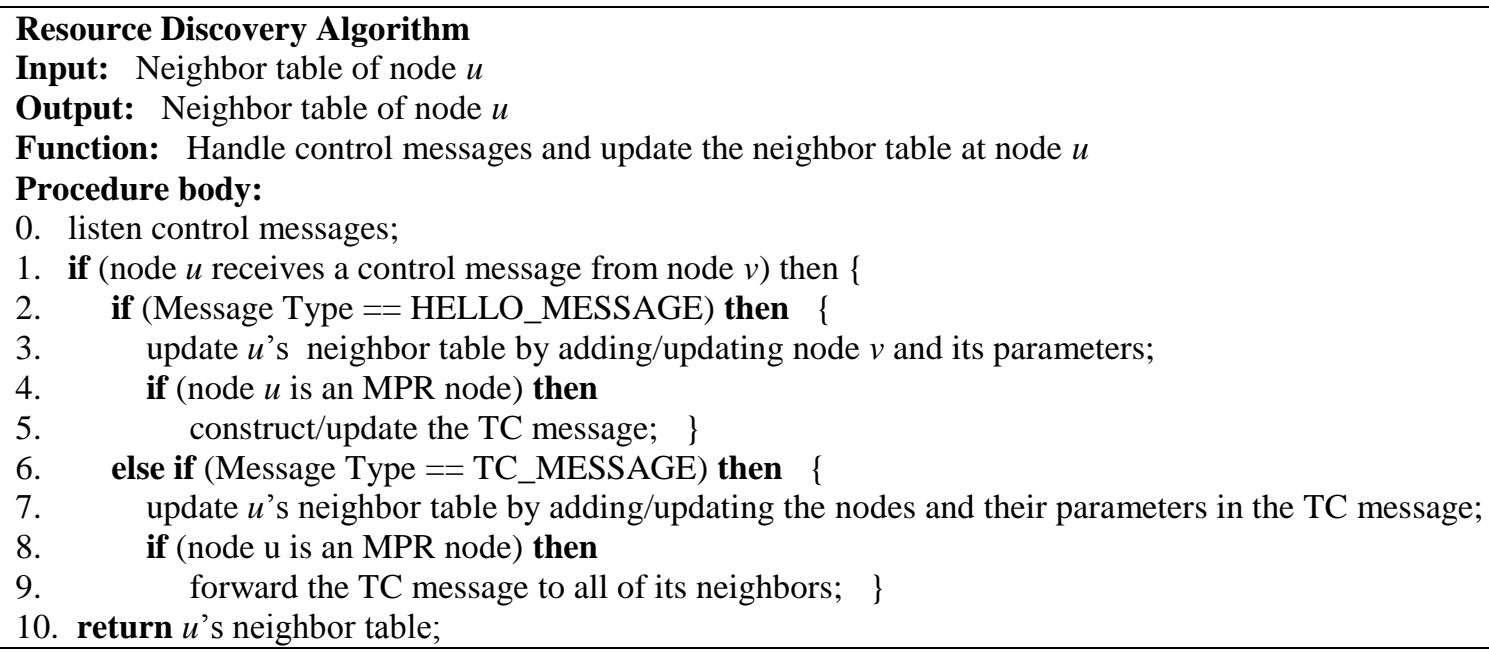

\section{Fig.1 Resource discovery algorithm}

\subsection{Phase II: Adaptive probabilistic scheduling phase}

Each time a source node $u$ receives a task $j$ submitted by its local user, it estimates the energy

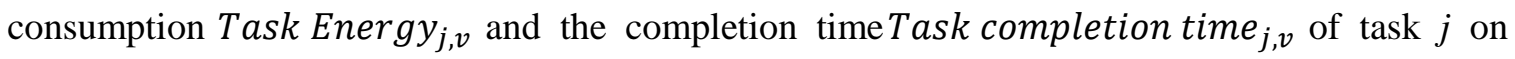
every potential processing node $v \in P$. These two variables are obtained according to Eqn. (4) and Eqn. (8), respectively.

$$
\text { Task completion } \text { time }_{j, v}<T_{j}-t_{\text {margin }}
$$

The scheduler keeps a set $P^{\prime}$ of processing nodes which satisfy Eqn. (15). The scheduler will randomly choose one processing node from the set. The probability that node $v \in P^{\prime}$ is chosen to be the processing node is

$$
\rho_{v}=\left(1 / \text { Task Energy }_{j, v}\right) / \sum_{v \in P^{\prime}}\left(1 / \text { Task Energy }_{j, v}\right)
$$

In a dynamic wireless environment, there is no guarantee that the tasks will be able to complete before the deadline. The reasons could be: 1) it is still possible that multiple source 
nodes schedule tasks to one processing node at the same time. Due to the queuing delay at that processing node, the actual execution time will be longer than the estimated completion time, causing the task unable to be completed within its time constraint; 2) the bandwidth may be shared among multiple transmissions so that Eq. no (7) cannot give an accurate transmission time.

When failure happens, the scheduler will increase its $t_{\text {margin }}$ value to avoid continuous future failures. When $a_{1}$ consecutive failed tasks occur, the time margin will be increased by $\Delta t_{1}$. After receiving $a_{2}$ consecutive successful tasks, the scheduler will decrease the time margin by $\Delta \mathrm{t}_{2}$. We initialize $a_{1}$ and $a_{2}$ as 2 in our simulations, which means when 2 consecutive failed tasks occur, the scheduler tends to choose the more powerful processing node at the cost of increasing energy consumption, and vice versa. We initialize $\Delta t_{1}$ and $\Delta \mathrm{t}_{2}$ as $0.05 \mathrm{~s}$ in our simulations. $\Delta t_{1}$ and $\Delta \mathrm{t}_{2}$ are related to the tasks' time constraints. We expect that $\Delta t_{1}$ and $\Delta \mathrm{t}_{2}$ will be increased with a longer time constraint. All these 4 parameters reflect how fast the adaptive probabilistic scheduler reacts to the failed tasks and they can be optimized. For example, if the scheduler aims at ensuring the task completion rate, it will adopt a small value of $a_{1}$ and a large value of $\Delta t_{1}$. The adaptive probabilistic scheduling algorithm running at each source node is given in Fig. 2. Assume that each arriving task will be placed into the task queue $J$ with its starting time $t \_s t a r t$ recorded.

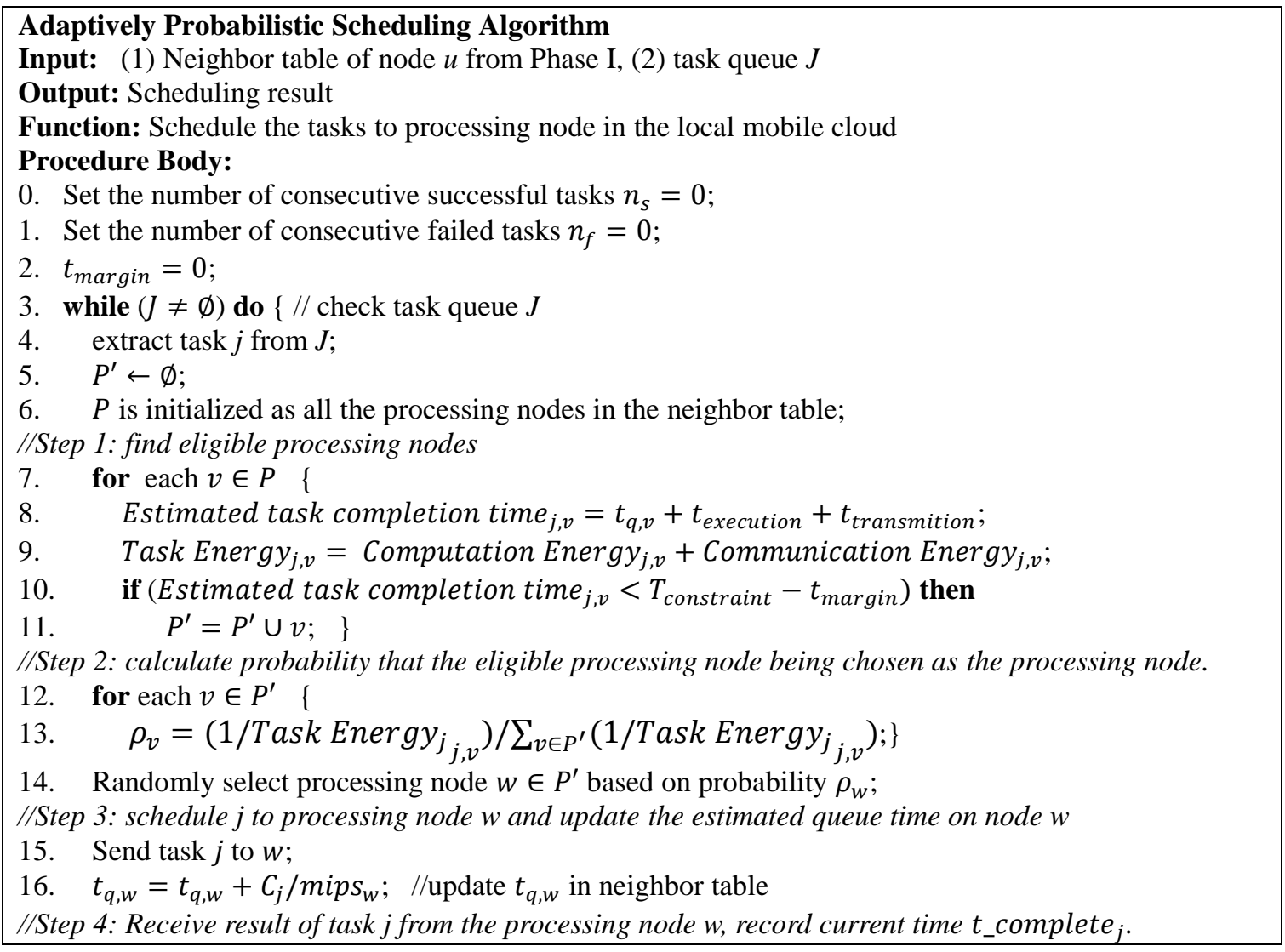




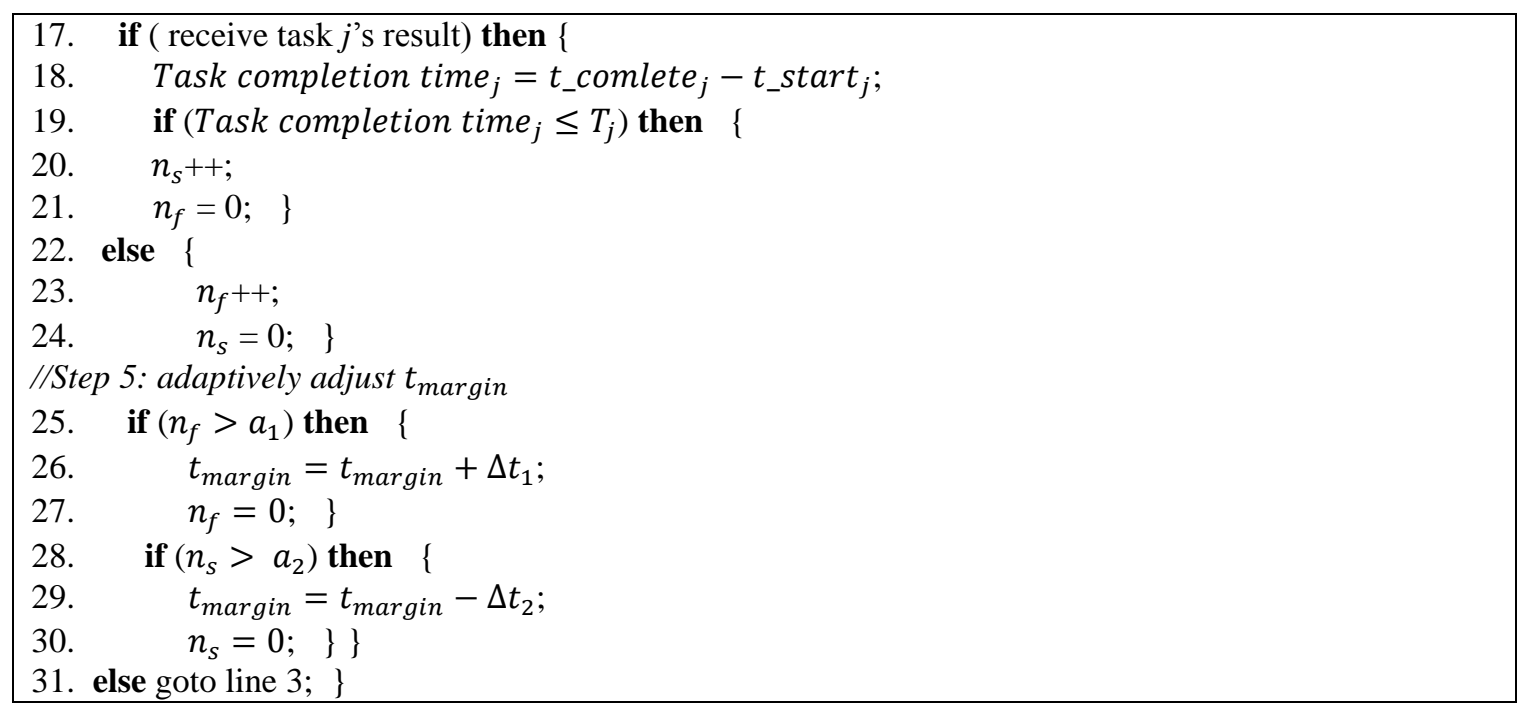

Fig. 2 Adaptively probabilistic scheduling algorithm

In this paper, the proposed scheduling algorithm is compared with three other scheduling schemes introduced in [12][26], including the round robin scheduler, the greedy scheduler, and the probabilistic scheduler. The round robin scheduler assigns tasks in a round robin way. When the first task arrives, it is scheduled to the first node in $P$. It schedules the second task to the second node in $P$, and so on. The greedy scheduler always sends the task to the most energy efficient node with the estimated completion time within the time constraint. The probabilistic scheduler keeps a set $P^{\prime}$ of the processing nodes satisfying Task completion time $e_{j, v}<T_{j}$. The scheduler will randomly choose one processing node from the set $P^{\prime}$. The probability that node $v \in P^{\prime}$ is chosen to execute the task is calculated by applying Eqn. (16).

Note that the round robin scheduler does not rely on the real-time resource information to make scheduling decisions, but uses basic OLSR protocol with long interval TC messages to collect the list of processing nodes. As mentioned in Section 2, most existing greedy schedulers in local mobile clouds didn't consider the resource information. However, for comparison purpose, here we use the same proposed resource discovery scheme for the rest three schedulers.

\subsection{An illustrative example}

We use Fig. 3 as an example to demonstrate the difference between different schedulers. Related parameters are listed in Table 2. This example is derived based on a speech recognition application [7]. There are two source nodes, s0 and s1, each streaming a 1s utterance of 8-bit, 4$\mathrm{kHz}$ speech request (referred as a task with 4000 bytes data size) to one of the four processing nodes, n0, n1, n2, and n3. Assume the channel bandwidth is up to $11 \mathrm{Mbps}$. 


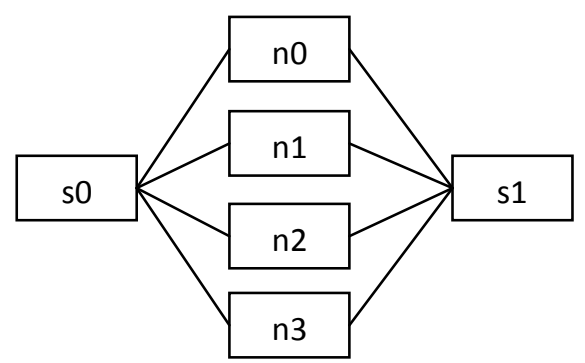

Fig. 3 A sample local mobile cloud for the illustrative example

Table 1. Parameter settings of the illustrative example

\begin{tabular}{ll}
\hline Parameter & Value \\
\hline Task arrival intervals & $0.5 \mathrm{~s}$ \\
Task data size & 4000 bytes \\
Task computation amount & 200 million instructions (MI) \\
Task time constraint & $1 \mathrm{~s}$ \\
$e_{n 0} / e_{n 1} / e_{n 2} / e_{n 3}$ & $0.25 / 0.16 / 0.09 / 0.04 \mathrm{~J} / \mathrm{MI}$ \\
mips $_{n 0} /$ mips $_{n 1} /$ mips $_{n 2} /$ mips $_{n 3}$ & $500 / 400 / 300 / 200 \mathrm{MI} / \mathrm{s}$ \\
\hline
\end{tabular}

At the start time $t=0 \mathrm{~s}$, two source nodes $\mathrm{s} 0$ and $\mathrm{s} 1$ start receiving tasks. There is one task arriving at each source node every $0.5 \mathrm{~s}$. For the proposed adaptive probabilistic scheduler, $t_{\text {margin }}$ is initialized to $0 \mathrm{~s}$. If one task is unsuccessfully scheduled $t_{\text {margin }}$ is increased by $0.5 \mathrm{~s}$; otherwise, $t_{\text {margin }}$ is decreased by $0.5 \mathrm{~s}$.

Since the communication energy is very small comparing to the computation energy, we ignore the communication energy in this example. We also ignore the transmission time for simplicity. According to Eq. no (5), the energy consumption for executing the task at the processing nodes $\mathrm{n} 0, \mathrm{n} 1, \mathrm{n} 2$ and $\mathrm{n} 3$ is $50 \mathrm{~J}, 32 \mathrm{~J}, 18 \mathrm{~J}$ and $8 \mathrm{~J}$, respectively.

Fig. 4 shows the scheduling results of four schedulers. Tasks from s0 are represented in black and s1 in red. Solid line is the execution time while dashed line is the waiting time at the processing node. The length of each line is the completion time of the task. If the duration of the line is longer than $1 \mathrm{~s}$, the task is failed. The completion rate and the energy per successful task are listed for each scheduler. Two source nodes always schedule tasks to the same processing node using the round robin scheduler and the greedy scheduler. The round robin scheduler arranges tasks to more powerful nodes (n0, n1), causing a high task completion rate as well as high energy consumption. The greedy scheduler always assigns tasks to the most energy efficient node satisfying time constraint. Therefore, a long task queue is easily present, which explains that the greedy scheduler has the lowest task completion rate. Two source nodes schedule tasks to different processing nodes using the probabilistic scheduler randomly based on Eq. (16). Comparing with the greedy scheduler, in terms of the number of tasks assigned to each processing node, the probabilistic scheduler only has one more task scheduled to $n 1$ instead of $n 3$. 
However, the performance improves a lot using the probabilistic scheduler. The adaptive probabilistic scheduler increases $t_{\text {margin }}$ at $t=1.5 \mathrm{~s}$ when the first failed task has been detected. For $\mathrm{s} 1, \mathrm{n} 2$ no longer satisfies the time constraint in Eq. (15). Thus at $t=1.5 \mathrm{~s}, \mathrm{~s} 1$ schedules task to n1. By doing this, the adaptive scheduler successfully avoids one failed task. The average energy per successful task is further improved.

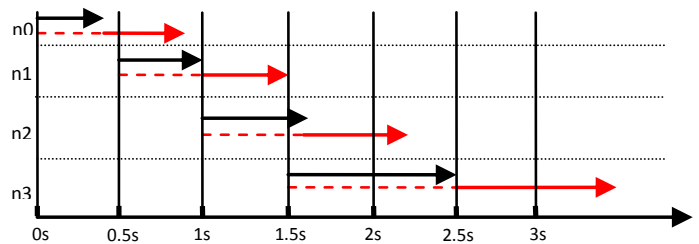

(a) Round Robin

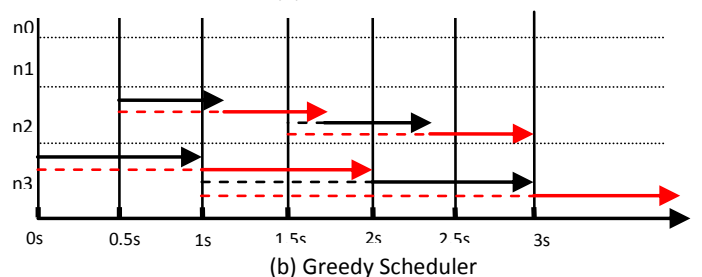

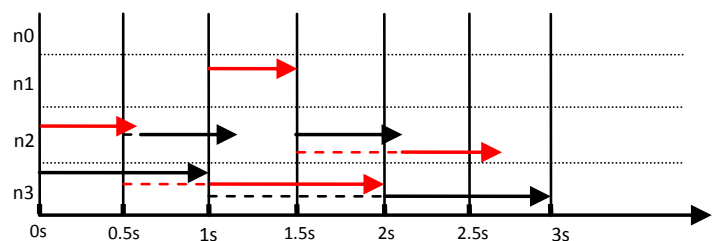

(c) Probabilistic Scheduler

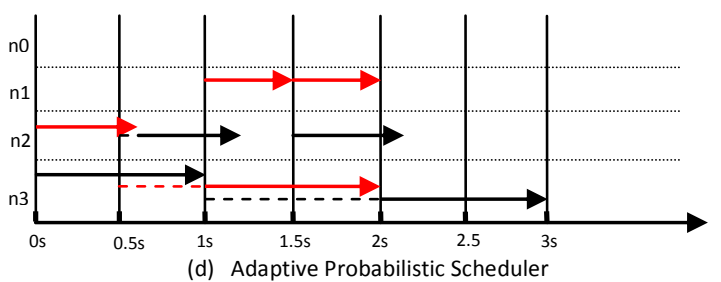

Fig. 4 Results of the illustrative example. (a) Round Robin Scheduler: Completion Rate: 75\%, Energy per successful task: 36 J/task, (b) Greedy Scheduler: Completion Rate: 37.5\%, Energy per successful task: 34.6 J/task, (c) Probabilistic Scheduler: Completion Rate: 62.5\%, Energy per successful task: 25.6 J/task (d) Adaptive Probabilistic Scheduler: Completion Rate: 75\%, Energy per successful task: $23.6 \mathrm{~J} /$ task

\section{Simulations and results}

To evaluate the performance of various scheduling algorithms in local mobile clouds, extensive simulations are conducted on a local mobile cloud simulation module developed on OMNET++ [32].

\subsection{Simulation setup}

The simulated local mobile cloud contains a group of nodes, each of which is capable of transmitting radio signals up to approximately 40 meters over an $11 \mathrm{Mbps} 802.11 \mathrm{~g}$ wireless channel. Three network scenarios (with different node densities) are simulated. The first scenario, referred to as the small network, is created by randomly placing 10 nodes in a $200 \mathrm{~m} \times 200 \mathrm{~m}$ area. Among these 10 nodes, 2 nodes are source nodes and 8 nodes are processing nodes. The second scenario, referred to as the medium network, is created by randomly placing 20 nodes in a $200 \mathrm{~m} \times 200 \mathrm{~m}$ area. Among these 20 nodes, 4 nodes are source nodes and 16 nodes are processing nodes. Similarly, in the large network, there are 8 source nodes and 32 processing nodes in the same area. Tasks are generated at each source node. The task arrival event is a Poisson process. Source nodes cannot be chosen to be the MPR nodes. The total data size varies 
from 2000 bytes to 16000 bytes. In our experiments, we assume that half data is sent from the source node to the processing node and the other half is sent back after the task is executed. Other parameters are listed in Table 3.

Table 3. Parameter settings in simulation

\begin{tabular}{|c|c|}
\hline Parameter & Value \\
\hline Topology & Random \\
\hline Network area & $200 \mathrm{~m} * 200 \mathrm{~m}$ \\
\hline Network size & Small/Medium/Large \\
\hline Communication range & Approximately 40 meters \\
\hline Communication power & $50 \mathrm{~nJ} / \mathrm{bit}[35]$ \\
\hline Computation ability of node $u \operatorname{mips}_{u}$ & Normal distribution in $(1000,300)$ mips \\
\hline Computation energy per MI of node $u$ & $10^{-8} \times \operatorname{mips}_{u}{ }^{2} \mathrm{~J}[34]$ \\
\hline Maximum bandwidth & $11 \mathrm{Mbps}$ \\
\hline Task data size & Varying from 2000 bytes to 16000 bytes \\
\hline Task computation amount & Varying from $50 \mathrm{MI}$ to $350 \mathrm{MI}$ \\
\hline Task time constraint & $0.5 \mathrm{~s}$ \\
\hline Task arrival interval & Exponential distribution $\lambda=0.2 \mathrm{~s}$ \\
\hline Simulation duration & $200 \mathrm{~s}$ \\
\hline
\end{tabular}

There are two mobility patterns: the first one is the stationary network in which all nodes are stationary. The second one is the mobile network in which every mobile node $u \in V$ moves within the area according to the following pattern. It moves along a straight line for a certain period of time before it makes a turn. This moving period is a random number, normally distributed with an average of 5 seconds and standard deviation of 0.1 second. When it makes a turn, the new direction (angle) in which it will move is a normally distributed random number with average equal to the previous direction and standard deviation of 30 degrees. Its speed is also a normally distributed random number ranging from 1 to $3(\mathrm{~m} / \mathrm{s})$. A new such as random number is picked as its speed when it makes a turn. All nodes can move to a random direction within the area with a speed uniformly distributed between $1 \mathrm{~m} / \mathrm{s}$ and $3 \mathrm{~m} / \mathrm{s}$ [4][6]. All nodes have the same initial battery level. The energy of a node is only consumed when the node processes a task or when the node transmits/receives a message, which can be calculated using Eq. (2)-(4). The energy consumed in moving is ignored here.

In the experiments, the performance of the proposed task scheduling scheme is compared with the other three scheduling algorithms in terms of the following metrics.

- Task completion rate: the ratio of the number of tasks finished within time constraint to the total number of tasks in Eq. (10).

- Average task waiting time: the average duration between a task's arrival time at the processing node and its execution start time at the processing node.

- Average energy per successful task: the ratio of the total energy consumption to the number of successful tasks given in Eq. (11).

\subsection{Simulation results}


In each experiment, 10 different random network topologies are generated. The simulation results are plotted using the average values derived from these 10 simulations.

\subsubsection{Effect of varying topology control message interval}

As introduced in Section 3, the QoS OLSR is modified by adding extra bytes to control messages. The influence of the modification is going to be evaluated in this section. In the original QoS OSLR, by default the Hello message interval is $2 \mathrm{~s}$ and TC message interval is $5 \mathrm{~s}$. In the modified QoS OLSR scheme, the Hello message interval is half of the TC message interval, guaranteeing that MPR nodes send TC message to the whole network with their neighbor information updated at least once. Considering the tight time constraints $(0.5 \mathrm{~s})$, we expect the control messages to be more frequently sent so that the resource information can be updated in time.

In order to gauge the overhead, we measure the number of bytes in the control messages, including HELLO messages and TC messages, in the small network and the large network, respectively. The average overhead traffic is defined as the number of bytes in the control messages transmitted per second in the whole network. The average overhead traffic shows how much network bandwidth is consumed by the overhead messages. Fig. 5 plots the average overhead traffic of the proposed modified QoS OLSR in the small network and the large network and the original QoS OLSR in the small network vs. varying TC message interval.

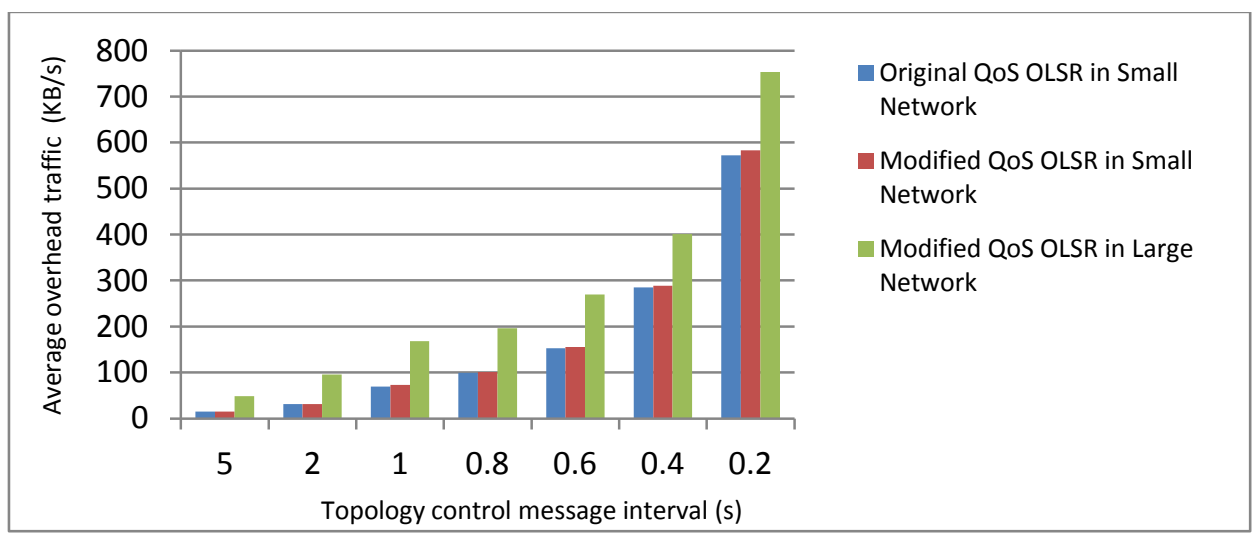

Fig. 5 Average overhead traffic

The average overhead traffic increases almost exponentially as TC messages get more frequent, when the TC message interval reaches $0.2 \mathrm{~s}$, the traffic is nearly $600 \mathrm{~KB} / \mathrm{s}$ in the small network and $750 \mathrm{~KB} / \mathrm{s}$ in the large network. In the small network, the traffic of modified QoS OLSR increases only slightly comparing to the original QoS OLSR (less than 2\%). The reason is that only 4 bytes are added to each Hello Message. In the TC message, though 4 bytes are added to each neighbor node, the neighbor list in TC messages is quite limited in a small network. Fig. 5 also shows that in the large network, there is more overhead traffic than in the small network. 
Next we investigate the performance of different schedulers under different TC message intervals in a small network with 2 source nodes and 8 processing nodes. As mentioned in Section 4.2, the TC interval for the round robin scheduler is set to be 2s for all cases. Fig. 6 shows that except the round robin scheduler, the task completion rates of the other three schedulers first increase as the TC message interval gets a shorter but decrease significantly when the TC message interval reaches $0.2 \mathrm{~s}$. The reason is that with a long TC message interval, there are not enough control messages carrying resource information, the schedulers are not able to get an accurate picture of nearby nodes. When control messages become more frequent, the resource information is updated at source nodes more frequently. As such, better scheduling results are obtained: the task completion rate increases. However, when the overhead messages occupy a large amount of network bandwidth (at TC interval of 0.2s), schedulers are unable to predict the accurate transmission time at the target processing node as in Eqn. (7). The scheduling results are significantly impacted, especially for greedy and probabilistic schedulers, which result in a dramatic drop of the task completion rates.

The round robin scheduler does not rely on the real-time resource information. It sends tasks to nearby nodes one after another. Thus its task completion rate remains the same. The proposed adaptive probabilistic scheduler has the highest completion rate for most cases. It has $16.6 \%$ and $3.6 \%$ higher task completion rate than the greedy scheduler and the probabilistic scheduler, respectively. The reason is that with an increased $t_{\text {margin }}$, the scheduler will send tasks to a more computationally powerful node when failure happens. Thus it has better chance to complete tasks before the time constraint. This result is further explained by Fig. 7.

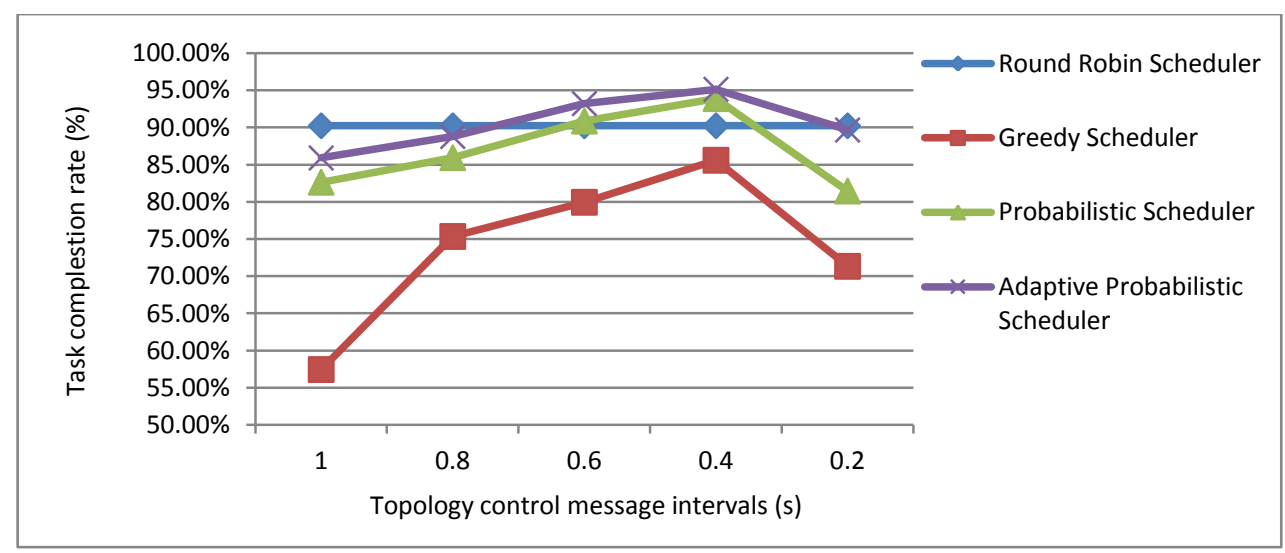

Fig. 6 Task completion rate vs. TC message interval in the small network

In Fig. 7, the simulation result shows that the average waiting time per task at the processing node of the greedy scheduler is much higher than other schedulers. It is because both source nodes always try to offload tasks to the most energy efficient node. A waiting queue is easily 
present in the target processing node, and the long waiting time causes the task unable to be completed before their deadlines. The probabilistic scheduler and the adaptive probabilistic scheduler alleviate this problem by randomly choosing the processing node to reduce the chance that multiple tasks are offloaded to the same processing node. When conflict does happen, the latter has a better chance to offload to a more powerful node with even lower average waiting time, which is 2-4 times shorter than the greedy scheduler. The round robin scheduler has the shortest waiting time because it sends the tasks to nearby processing node evenly.

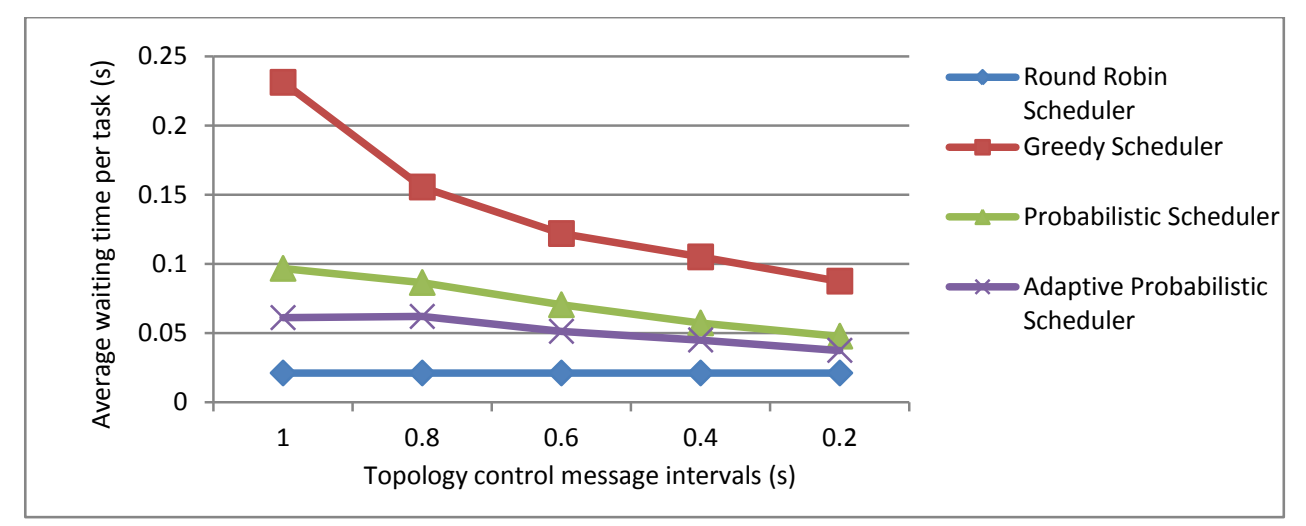

Fig. 7 Average waiting time per task vs. TC message interval in the small network

Fig. 7 also demonstrated how TC message interval affects the scheduler's performance. With a shorter TC message interval, more accurate resource information is achieved. Thus the schedulers are less possible to send the tasks to a node which already has long waiting queue.

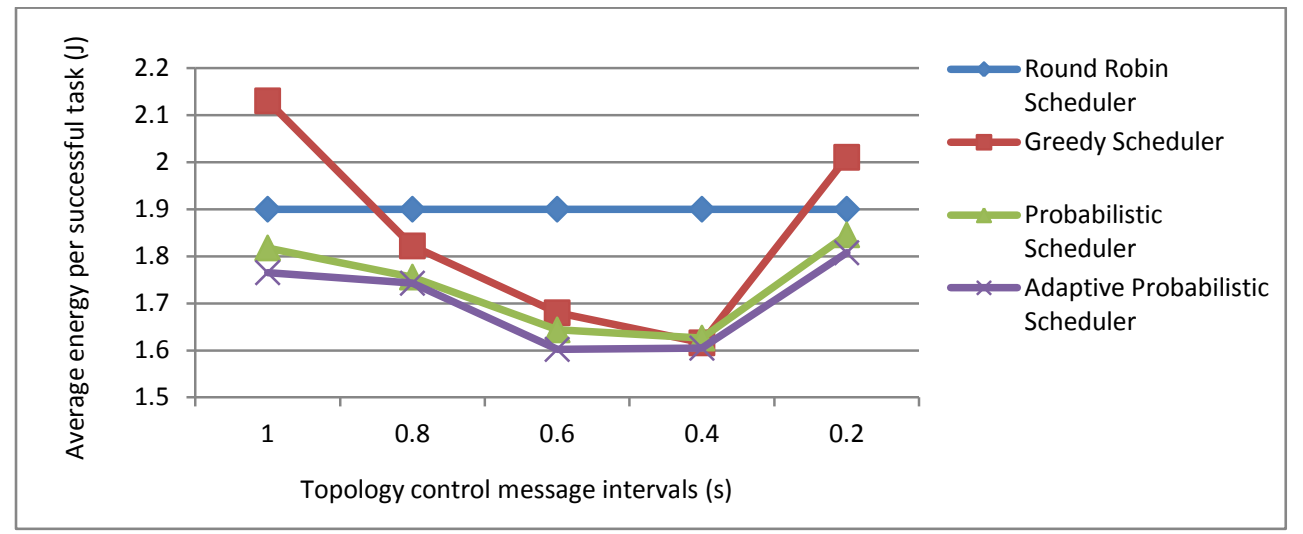

Fig. 8 Average energy per successful task vs. TC message interval in the small network

Fig. 8 shows that the average energy per successful task is correlated with the task completion rate. Except for the round robin scheduler, the average energy per successful task of the other three schedulers, first decreases as the TC message interval is reduced but increases significantly when the TC message interval reaches $0.2 \mathrm{~s}$. Among all four schedulers, the average energy per successful task of the adaptive probabilistic scheduler is the best. When the TC 
message interval is $0.4 \mathrm{~s}$, the adaptive probabilistic scheduler has the highest completion rate $95.14 \%$ and the lowest energy per successful task $1.60 \mathrm{~J}$.

Noticeably, the impact of the TC message interval is different for larger size networks. We repeat the same experiment for the large network. As shown in Table 4, the task completion rates of all four schedulers are lower than in a small network. The reason is that it takes longer time to transmit data from the source node to the processing node in the large network due to two main reasons as discussed in [35]: 1) Multi-hop delay: each intermediate node needs to receive the entire message then retransmit it to next hop. 2) Channel access delay: more nodes trying to send data at the same time in the network generates more collisions, which causes additional delay. With a shorter TC message interval, the overhead traffic is greater as shown in Fig. 5, which further impacts the transmission time. Therefore, the task completion rates of the other three schedulers except the round robin scheduler decrease as the TC message interval becomes shorter. Again, the proposed adaptive probabilistic scheduler has higher completion rate than greedy and probabilistic schedulers for all cases. Table 4 also shows that the average energy per successful task of the adaptive probabilistic scheduler is lower than the other three schedulers when TC message interval is greater than or equal to $0.6 \mathrm{~s}$. The adaptive probabilistic scheduler achieves a $15.36 \%$ reduction in the average energy per successful task comparing with the other three schedulers when the TC message interval is 1s. Except for the round robin scheduler, the average energy per successful task of the other three schedulers increase as the TC message interval becomes shorter due to the decreased task completion rate.

Table 4. Impact of TC interval to scheduling results in the large network

\begin{tabular}{|l|r|r|r|r|r|r|r|r|}
\hline & \multicolumn{4}{|c|}{ Task completion rate } & \multicolumn{3}{c|}{ Average energy per successful task } \\
\hline & $1 \mathrm{~s}$ & $0.8 \mathrm{~s}$ & $0.6 \mathrm{~s}$ & $0.4 \mathrm{~s}$ & $1 \mathrm{~s}$ & $0.8 \mathrm{~s}$ & $0.6 \mathrm{~s}$ & $0.4 \mathrm{~s}$ \\
\hline TC intervals & & & & & & & & \\
\hline $\begin{array}{l}\text { Round Robin } \\
\text { Scheduler }\end{array}$ & $74.34 \%$ & $74.34 \%$ & $74.34 \%$ & $74.34 \%$ & 2.498 & 2.498 & 2.498 & 2.498 \\
\hline Greedy Scheduler & $49.87 \%$ & $46.62 \%$ & $37.36 \%$ & $16.11 \%$ & 2.470 & 2.533 & 2.985 & 4.658 \\
\hline $\begin{array}{l}\text { Probabilistic } \\
\text { Scheduler }\end{array}$ & $49.19 \%$ & $47.73 \%$ & $43.27 \%$ & $27.26 \%$ & 2.501 & 2.451 & 2.390 & 3.163 \\
\hline $\begin{array}{l}\text { Adaptive } \\
\text { Probabilistic } \\
\text { Scheduler }\end{array}$ & $56.89 \%$ & $54.97 \%$ & $50.42 \%$ & $29.02 \%$ & 2.107 & 2.212 & 2.253 & 3.092 \\
\hline
\end{tabular}

\subsubsection{Impact of network size}

In this experiment, we are going to investigate the detailed influence of the size of the network on the performance of the schedulers. According to the results from previous experiment, we adopt $0.4 \mathrm{~s}$ TC message interval in the small/medium network and $1 \mathrm{~s}$ TC message interval is used in the large network. Fig. 9 shows that in a larger network, the task completion rate is lower. Comparing with the small network, the task completion rate is $5 \%$ lower on average for all four 
schedulers in the medium network and $33.65 \%$ in the large network. This is due to three reasons. First, the resource discovery scheme generates more overhead traffic in a large network as each node keeps broadcasting its resource information. Second, the large network requires more hops between the source and the potential processing node. At each hop, there could be a transmission queue which increases the unpredictability of the transmission time. Third, more source nodes increase the workload of the network as more tasks need to be scheduled at the same time, which makes the efficient scheduling even harder.

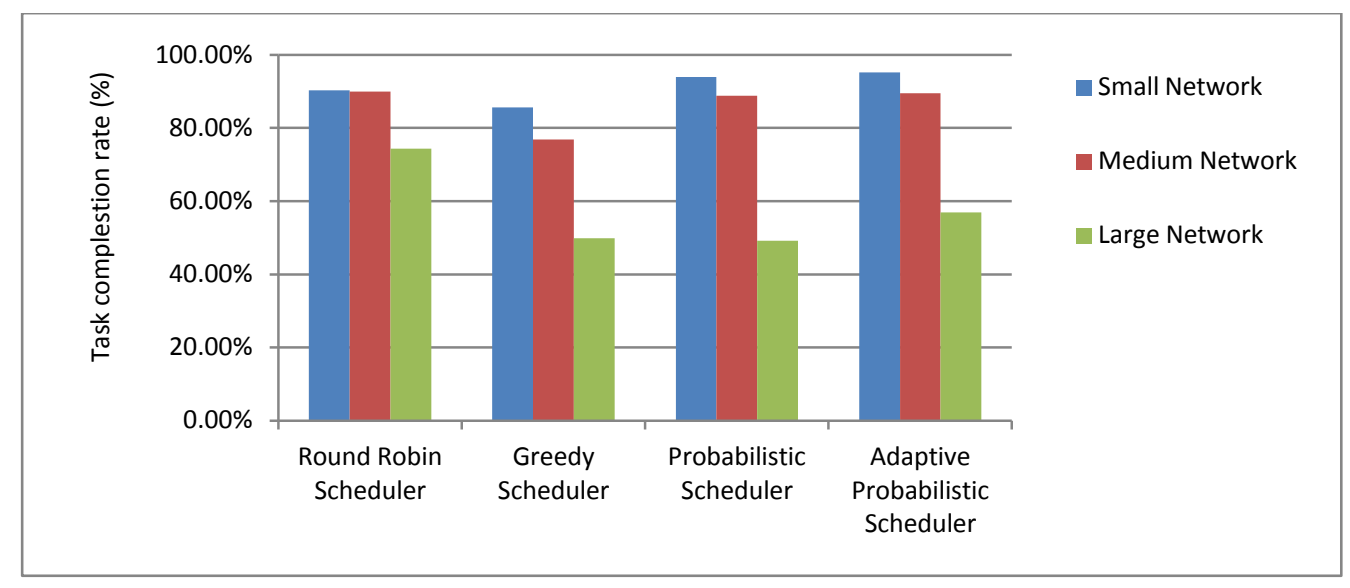

Fig. 9 Task completion rate vs. network size

In Fig. 10, the average energy per task is $12.7 \%$ higher on average for all four schedulers in the medium network and $42.3 \%$ in the large network, comparing with the small network. The main reasons are greater communication energy caused by increased hop counts as well as increased overhead traffic, and decreased task completion rate. The advantage of the adaptive probabilistic scheduler in the large network is more significant. Comparing with other schedulers, the adaptive probabilistic scheduler reduced $18.1 \%$ energy per successful task on average in the large network while $17.4 \%$ in the medium network and $6.8 \%$ in the small network. The reason is that there are more energy efficient nodes in the large network. Thus the adaptive probabilistic scheduler can adjust its parameter to choose a target processing node from a larger set of processing nodes to avoid conflict. 


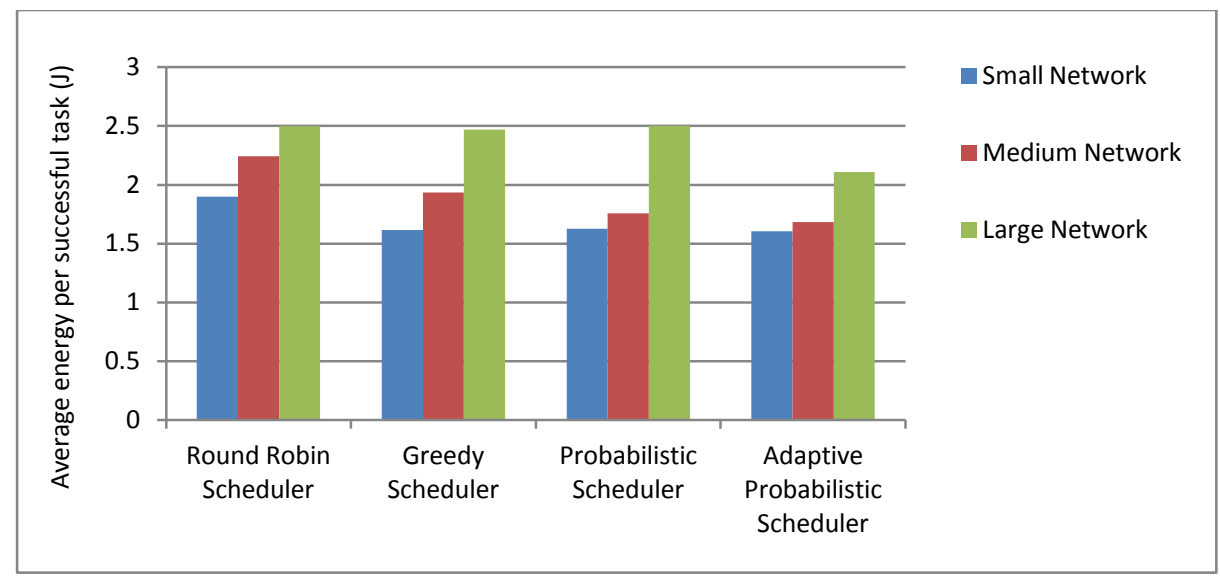

Fig. 10 Average energy per successful task vs. network size

\subsubsection{Impact of node mobility}

This experiment shows how the performance is affected by the mobility of the small network with the TC as $0.4 \mathrm{~s}$. Fig. 11 shows that the task completion rates of all schedulers are lower in the mobile network than in the stationary network. The round robin scheduler has an $4.66 \%$ lower task completion rate in the mobile network than in the stationary network while the other three schedulers only have $3.34 \%$ decrease on average. The reason is that mobility increases the context change, causing difficulty to gather accurate resource information. The resource discovery scheme used by the other three schedulers except the round robin scheduler updates the resource information periodically, increasing the chance of gathering the accurate resource information even when the nodes are moving. Again, the adaptive probabilistic scheduler has the highest task completion rate in both stationary and mobile networks due to its ability to adjust to the changing context.

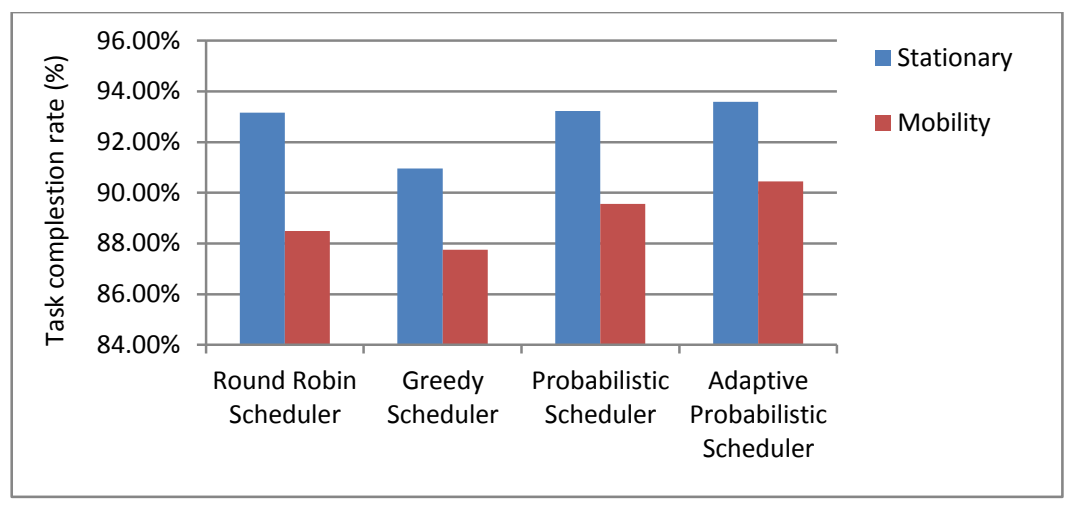

Fig. 11 Task completion rate in stationary and mobile network

Fig. 12 gives an inconsistent result regards to the average energy per successful task. The round robin scheduler consumes $2.95 \%$ more energy per successful task in the mobile network than in the stationary network. The greedy scheduler consumes $1.29 \%$ less. The probabilistic scheduler consumes $0.56 \%$ more energy in the mobile network. The adaptive probabilistic scheduler consumes almost the 
same amount of energy in both networks. This result in Fig. 12 shows that the average energy per successful task is not heavily affected by the node mobility as to the task completion rate.

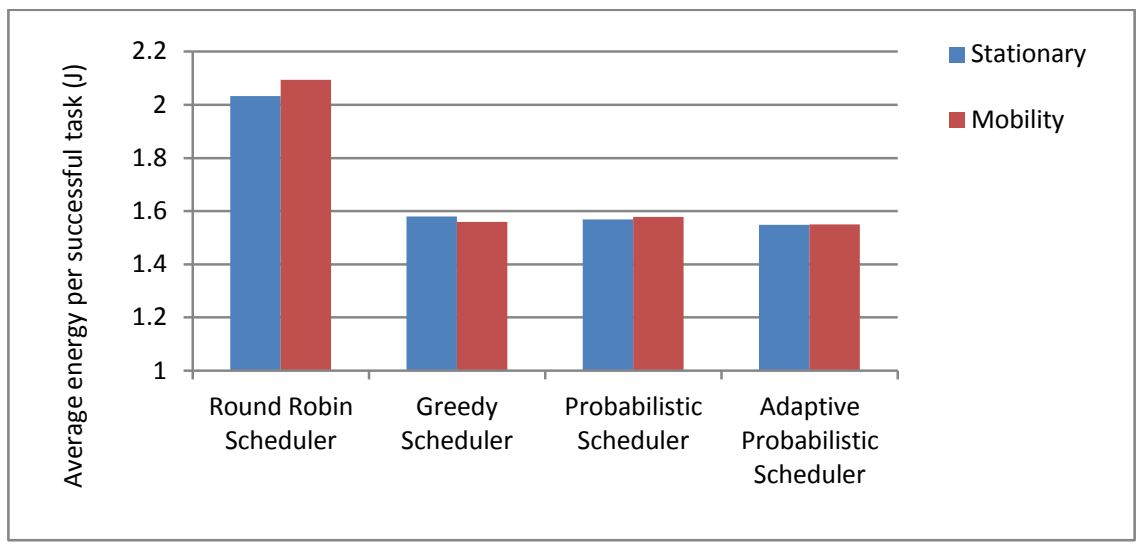

Fig. 12 Average energy per successful task in stationary and mobile network

\subsubsection{Impact of different task types}

In this experiment, we investigate the performance of these four schedulers with different task type in the small network. First, we fix the task data size then vary the computation amount. There are three workload scenarios shown in Table 5: low, medium, and high workload. In low workload case, the task computation amount is uniformly distributed between 50 to 150 million instructions. In medium and high workload cases, the task computation amount in uniformly distributed between 150 to 250 million instructions and between 250 to 350 million instructions, respectively. The computation amounts are selected according to the computation load of typical real-time application tasks, such as the motion detection and object recognition algorithms [6].

Table 5. Three different workload scenarios

\begin{tabular}{ccc}
\hline & $\begin{array}{c}\text { Average computation amount } \\
\text { (million instructions) }\end{array}$ & $\begin{array}{c}\text { Data size } \\
\text { (bytes) }\end{array}$ \\
\hline Low workload & 100 & 2000 \\
Medium workload & 200 & 2000 \\
High workload & 300 & 2000 \\
\hline
\end{tabular}

Fig. 13 shows that the task completion rate drops with the increasing workload for all four schedulers. The task completion rate of the round robin scheduler drops abruptly under heavy workload. The reason is that the round robin scheduler does not check the resource information. This impacts the scheduling results for tasks with a heavy computation amount which require more computation time at processing nodes. The greedy scheduler drops linearly. The probabilistic scheduler and the adaptive probabilistic scheduler drop more slowly. 


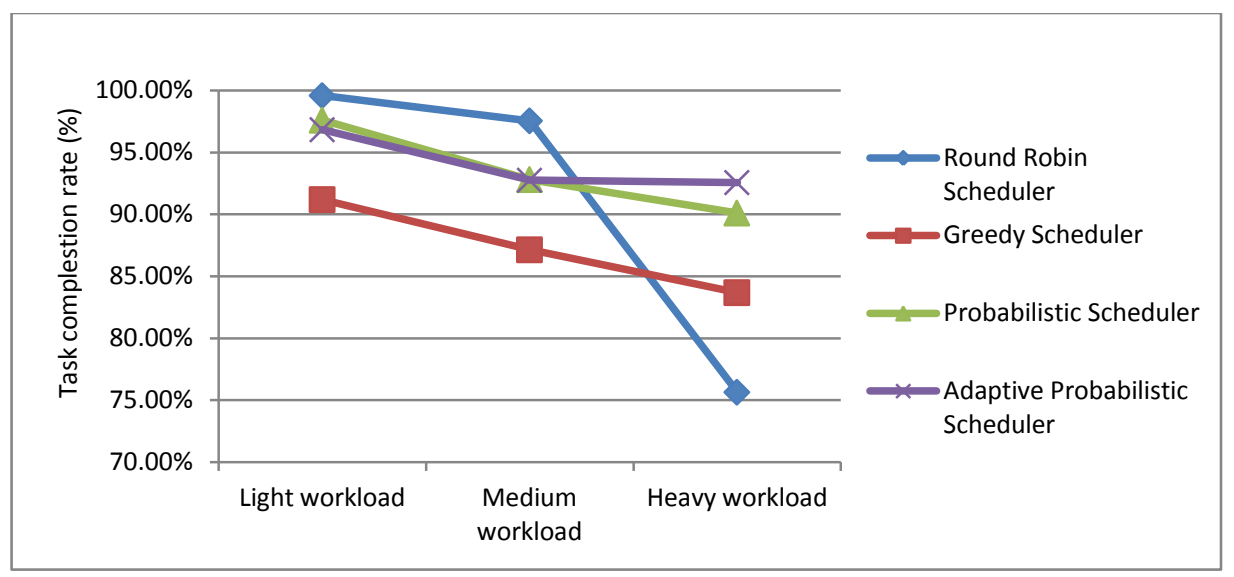

Fig. 13 Task completion rate in different workload scenarios

Fig. 14 shows that the average energy per successful task increases for all four schedulers. The average energy per successful task of the round robin scheduler in heavy workload scenario is 4.33 times as in low workload scenario. The average energy per successful task of the other three schedulers in heavy workload scenario is over 8 times as in low workload scenario. It is related to the square relation between the computational energy consumption and computation size in Eqn. (1).

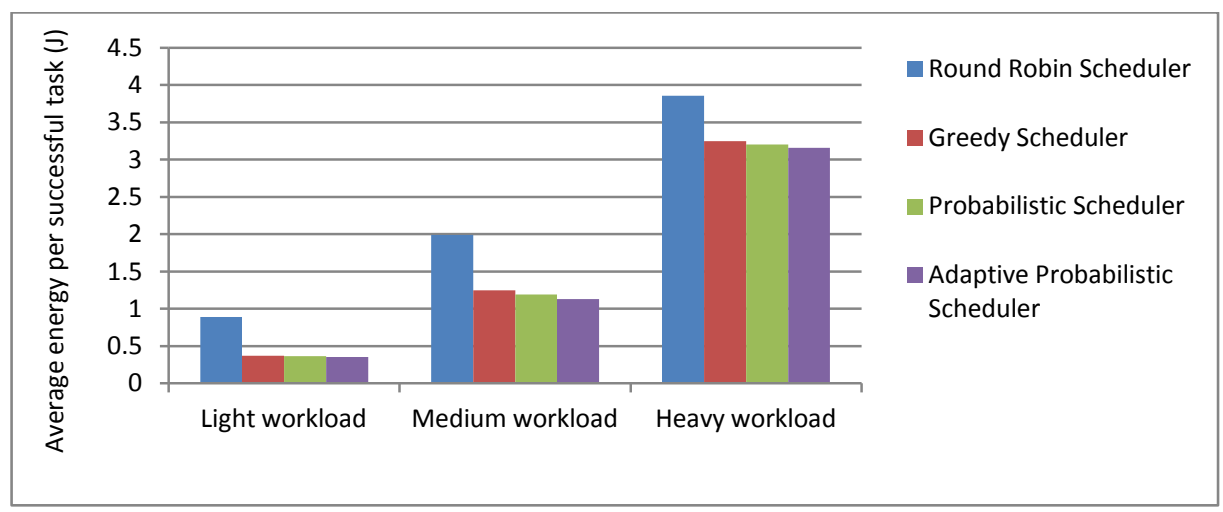

Fig. 14 Average energy per successful task in different workload scenarios

Next we vary the task data size and fix the computation amount. There are three types of data size as shown in Table 6: small, medium, and large data size. In small data size case, the task data size is uniformly distributed between 1000 to 3000 bytes. In medium and high workload cases, the task computation amount in uniformly distributed between 7000 to 9000 bytes and between 15000 to 17000 bytes, respectively. The medium and high data sizes reflect the data size needed for applications such as speech recognition [7] and object recognition [6].

Table 6. Three different data size scenarios

\begin{tabular}{ccc}
\hline & $\begin{array}{c}\text { Average data size } \\
\text { (bytes) }\end{array}$ & $\begin{array}{c}\text { Average computation amount } \\
\text { (million instructions) }\end{array}$ \\
\hline Small data & 2000 & 100 \\
Medium data & 8000 & 100 \\
\hline
\end{tabular}




\begin{tabular}{lll}
\hline High data & 16000 & 100 \\
\hline
\end{tabular}

Fig. 15 shows that the task completion rate drops when data size increases. All four schedulers have a similar trend. The reason is with a larger data size, tasks need to wait longer time for other tasks finish transmitting because of the shared transmission medium. The inaccuracy of the estimated transmission time in Eqn. (7) increases. From the medium data size to the large data size, the task completion rate of the adaptive probabilistic scheduler only decreases by $9.8 \%$. The task completion rate of the round robin scheduler, the greedy scheduler and the probabilistic scheduler decreases by $12.1 \%, 16.5 \%$ and $12.1 \%$, respectively.

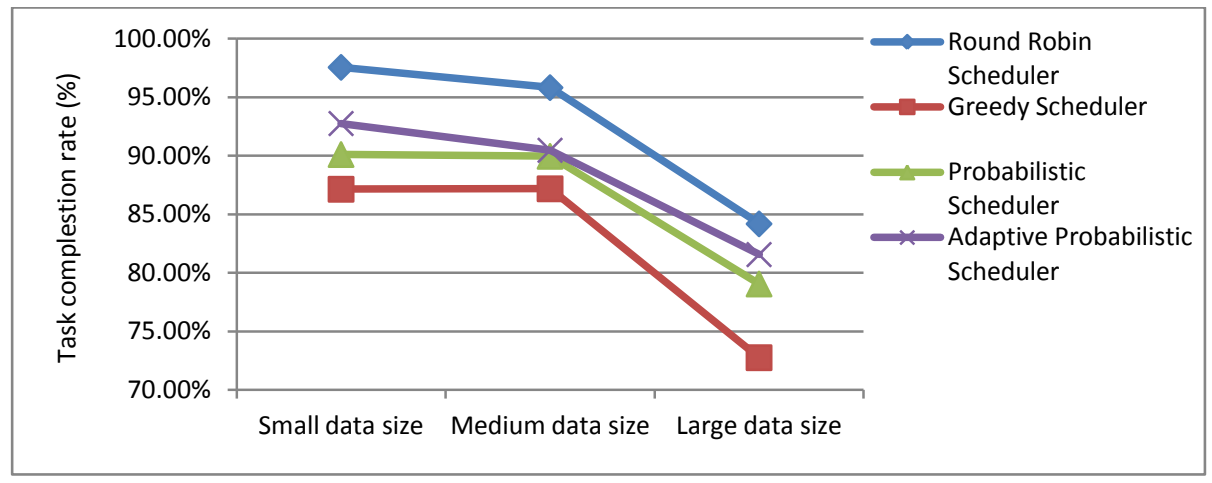

Fig. 15 Task completion rate vs. different data size

Fig. 16 shows that with increasing data size, the average energy per successful task of all four schedulers is also increased. This is attributed to the decreased task completion rate and the increased communication energy. From the small data size to the large data size, the average energy per successful task of the round robin scheduler is increased 13.87\% from small data size to large data size. The greedy scheduler, the probabilistic scheduler and the adaptive probabilistic scheduler have increased of $20.53 \%, 23.07 \%$ and $25.66 \%$, respectively. The adaptive probabilistic scheduler has the greatest energy increase because it tends to choose the more powerful processing nodes to improve the task completion rate when failed tasks occur, which explains why in Fig. 15 the adaptive probabilistic scheduler has the smallest decrease in task completion rate. 


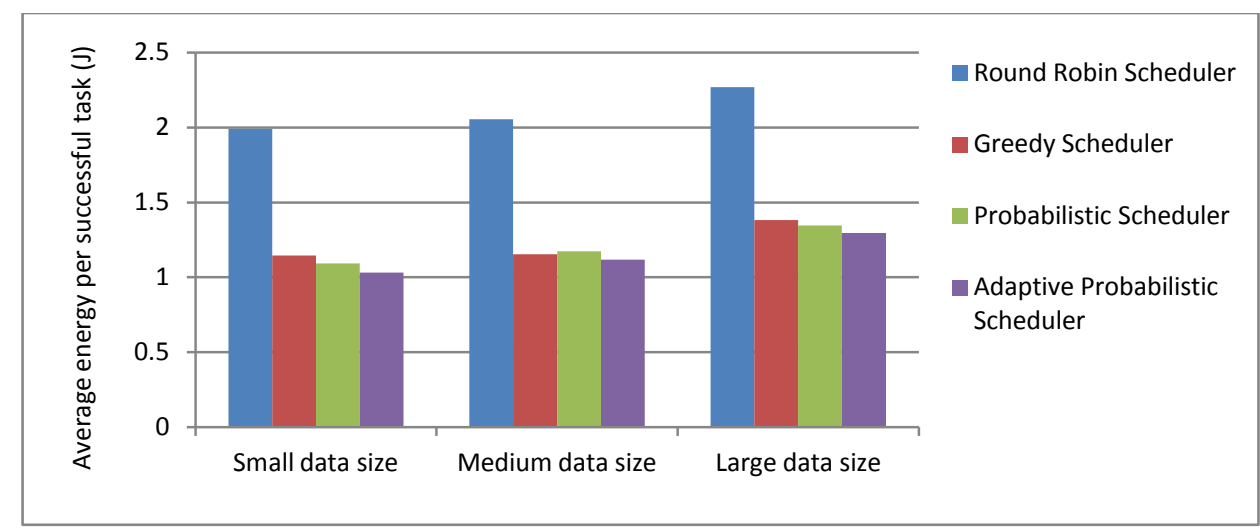

Fig. 16 Average energy per successful task vs. different data size

\subsection{Summary}

Firstly, we show that in the small network, by providing more frequent control messages the improvement is obtained for all the schedulers except the round robin scheduler. Then we show that in the large network, task completion rate is generally lower but the proposed scheduler can save more energy. We also find that though mobility degrades all schedulers' performance, the proposed scheduler achieves the highest completion rate with the lowest energy consumption. In summary, the round robin scheduler does not rely on the resource information. It assigns tasks evenly to all nearby processing nodes, yielding high completion rate but high energy consumption. The greedy scheduler suffers from low task completion rate because multiple tasks tend to be scheduled to the same processing node. The probabilistic scheduler provides higher task completion rate and lower average energy per task compared to the greedy scheduler because it reduces the possibility that more than one task is assigned to the same processing node. The adaptive probabilistic scheduler further improves the task completion rate by dynamically adjusting the set of potential eligible processing nodes. In most cases, it achieves the highest task completion rate and the lowest average energy per successful task with different types of tasks, which makes it an efficient task scheduler in local mobile clouds.

\section{Conclusion}

In this paper, we proposed an adaptive probabilistic task scheduler for scheduling real-time applications in local mobile clouds. In light of QoS OLSR, a source node receives periodical control messages to discover and update its nearby resource information. The scheduler first estimates the task completion time and energy consumption at each potential processing node based on the resource information collected through the modified QoS OLSR. Next, it schedules the current task to the proper processing node in a probabilistic way. Then it adaptively adjusts its time margin parameter to improve performance under the unpredictable network conditions. Overall, the observed experimental results confirm that the adaptive probabilistic scheduler can 
reduce the average energy per successful task while maintain a high task completion rate. Furthermore, the performance improvement becomes more noticeable when the number of source nodes increases. In addition, the proposed scheduler also shows high adaptability with different task types. This adaptive probabilistic scheduler is a viable approach for real-time applications due to its scalability and flexibility in a local mobile cloud.

Future work includes extending the proposed scheduler with more complex computation model at processing nodes, such as a parallel computing model with multiple cores. Furthermore, each task is only allowed to be scheduled once in the paper. Another work is to improve the scheduling performance by adding a rescheduling phase for tasks with high failure probability.

\section{Acknowledgements}

The work is supported in part by NSF under grant no. IIA-1301726, and fully supported by National Natural Science Foundation of China (No.61100004).

\section{References}

[1] A.R. Khan, M. Othman, S.A. Madani, S.U. Khan, A survey of mobile cloud computing application models, IEEE Communications Survey \& Tutorials, vol. 16, no. 1, pp. 393-412, Jan.-Mar. 2014.

[2] T. Verbelen, P. Simoens, D. Filip, D. Bart, Cloudlets: bringing the cloud to the mobile user, in: Proceedings of 3rd ACM Workshop on Mobile Cloud Computing and Services, Ambleside, UK, 2012, pp. 29-36.

[3] R. Kemp, N. Palmer, T. Kielmann, F. Seinstra, N. Drost, J.Maassen, H. Bal, EyeDentify: Multimedia Cyber Foraging from a Smartphone, in: Proceedings of 11th IEEE International Symposium on Multimedia(ISM), San Diego, USA, 2009, pp. 392-399.

[4] R. Fantacci, D. Tarchi, A. Tassi, A novel routing algorithm for mobile pervasive computing, in: Proceedings of Global Telecommunication Conferrence, Miami, USA, 2010, pp. 1-5.

[5] S. Shi, C. Hsu, K.Nahrstedt, R.Campbell, Using graphics rendering resources to enhance the real-time video coding for mobile cloud gaming, in: Proceedings of 19th ACM International Conference on Multimedia, Scottsdale, USA, 2011, pp. 103-112.

[6] Y. Nimmagadda, K. Kumar, Y. Lu, C.S.G. Lee, Real-time moving object recognition and tracking using computation, in: Proceedings of IEEE/RSJ International Conference on Intelligent Robots and Systems, Hilton Head Island, USA, 2010, pp. 2449-2455.

[7] R.C. Rose and I. Arizmendi, Efficient client-server based implementation of mobile speech recognition services, Speech Communication, vol. 48, pp. 1573-1589, 2006.

[8] K Smelcerz, Recent developments in mobile cloud scheduling: state-of-the-art, challenges and perspectives, Journal of Telecommunications and Information Technology, pp 51-57, Apr. 2013.

[9] S. Sotiriadis, N. Bessis, and N. Antonopoulos, Towards inter-cloud schedulers: a survey of metascheduling approaches, in: Proceedings of Int'I Conf. on P2P, Parallel, Grid, and Cloud and Internet Computing, Barcelona, 2011.

[10] E. Marinelli, Hyrax: cloud computing on mobile devices using MapReduce, Masters Thesis, Carnegie Mellon University, 2009.

[11] E.Cuervo, A. Balasubramanian, D. Cho, S. Saroiu, R. Chandra, P. Bahl, MAUI: making smartphones last longer with code offload, in: Proceedings of 8th International Conference on Mobile Systems, Applications, and Services, San Francisco, USA, 2010, pp. 49-62.

[12]X. Lu, H. Hassanein, S. Akl, Energy aware dynamic task allocation in mobile ad-hoc networks, in: Proceedings of International Conference on Wireless Networks Communications and Mobile Computing, Sheraton Maui Resort, USA, 2005, pp. 534-539. 
[13] S. Sindia, S. Gao, B. Black, A. Lim, V. Agrawal, P. Agrawal, MobSched: customizable scheduler for mobile cloud computing, in: Proceedings of 45th Southeastern Symposium on System Theory, Waco, USA, 2013, pp. 129-134.

[14] K. Christodoulopoulos, V. Sourlas, I. Mpakolas, E. Varvarigos, A comparison of centralized and distributed meta-scheduling architectures for computation and communication tasks in Grid networks, Computer Communications. 32 (7-10) (2009) 1172-1184.

[15] W. Alsalih, S. Akl, and H. Hassanein, Energy-aware task scheduling: towards enabling mobile computing over MANETs, in: Proceedings of IEEE Int'I Parallel and Distributed Processing Symp., 2005.

[16] D. Yao, C. Yu, H. Jin, and J. Zhou, Energy efficient task scheduling in mobile cloud computing, Lecture Notes in Computer Science, vol. 8147, pp. 344-355, 2013.

[17] X. Lin, Y. Wang, Q. Xie, P. M, Energy and performance-aware task scheduling in a mobile cloud computing environment, in: Proceedings of IEEE Int'I Conf. Cloud Computing (CLOUD), 2014.

[18] H. Eom, P. S. Juste, R. Figueiredo, Machine learning-based runtime scheduler for mobile offloading framework, in: Proceedings of IEEE/ACM $6^{\text {th }}$ Int'I Conf. Utility and Cloud Computing, 2013.

[19] R. Sen, G.-C. Roman, and C. Gill, Distributed allocation of workflow tasks in MANETs, Department of Computer Science and Engineering, Washington University in St. Louis, Report no. 2007-41, 2007.

[20] W. Alsalih, S. Akl, and H. Hassanein, Coperative ad hoc computing: towards enabling cooperative procesing in wireless environments, Int'l Journal of Parallel, Emergent and Distributed Systems, vol. 23, no. 1, pp. 59-79, Jan. 2008.

[21] H. Mukhtar, D. Belaid, G. Bernard, A graph-based approach for ad hoc task composition considering user perference model and device capabilities, in: Proceedings of Globecom workshiops, 2008.

[22] A. Bokar, M. Bozyigit, C. Sener, Scalable energy-aware dynamic task allocation, Advanced Information Networking and Application Workshops, in: Proceedings of Advanced Information Networking and Applications Workshops (WAINA), pp. 371-376, 2009.

[23] Y. Yang, X. Qiu, L. Meng, and L. Rui, A self-adaptive method of task allocation in clustering-based MANETs, in: Proceedings of Network Operations and Management Symp. (NOMS), 2010.

[24] C. Comito, D. Falcone, D. Talia, P. Trunfio, Energy efficient task allocation over mobile networks, in: Proceedings of IEEE $9^{\text {th }}$ Int'I Conf. Dependable, Autonomic and Secure Computing (DASC), 2011.

[25] M.C. Castro, A.J. Kassler, C.-F. Chiasserini, C. Casetti, I. Korpeoglu, Peer-to-peer overlay in mobile adhoc networks, Handbook of Peer-to-Peer Networkng, Springer, 2010.

[26] T. Soyata, R. Muraleedharan-Sreekumaridevi, C. Funai, M. Kwon, W. Heinzelman, Cloud-Vision: realtime face recognition using a mobile-cloudlet-cloud acceleration architecture, in: Proceedings of 17th IEEE Symposium on Computers and Communications, Cappadocia, Turkey, 2012, pp. 59-66.

[27]Y. Huang, N. Bessies, P. Norrington, P. Kuonen, B. Hirsbrunner, Exploring decentralized dynamic scheduling for grids and clouds using the community-aware scheduling algorithm, Future Generation Computer Systems. 29 (1) (2011) 402-415.

[28] V. Subramani, R. Kettimuthu, S. Srinivasan, P. Sadayappan, Distributed job scheduling on computational Grids using multiple simultaneous requests, in: Proceedings of 11th IEEE International Symposium on High Performance Distributed Computing, Edinburgh, UK, 2002, pp. $359-366$.

[29] H. Badis, K. Al Agha, QOLSR, QoS routing for ad-hoc wireless networks using OLSR, Wiley European Transactions on Telecommunications. 2005, 15(4), 427-442.

[30] M. Annavaram, E. Grochowski, J. Shen, Mitigating Amdahl's Law through EPI Throttling, in: Proceedings of 32nd Annual International Symposium on Computer Architecture, Madison, USA, 2005, pp. 298-309.

[31]T. Clausen, P.Jacquet, Optimized link state routing protocol, http://www.olsr.org, 2003.

[32] Omnet++ community site, http://www.omnetpp.org, 2013.

[33] K. Gomez, T. Rasheed, R. Riggio, D. Miorandi, C. Sengul, N. Bayer, Achilles and the Tortoise: Power consumption in IEEE 802.11n and IEEE 802.11g networks, Proceedings of IEEE Online Conference on Green Communications (GreenCom), 2013, pp. 20-26.

[34] E. Grochowski, M. Annavaram, Energy per instruction trends in Intel microprocessors, technology@Intel magazine. 4(3), (2006) 1-8. 
[35] R.V.P. Yerra, P. Rajalakshmi, Effect of relay nodes on end-to-end delay in multi-hop wireless ad-hoc networks, in: Proceedings of 27th International Conference on Advanced Information Networking and Applications Workshops (WAINA), Barcelona, Spain, 2013, pp. $343-348$. 